\title{
Sobre a ocorrência de Callistomys pictus (Pictet) (Rodentia, Echimyidae)
}

\author{
Sergio Maia Vaz ${ }^{1}$
}

\begin{abstract}
On the occurrence of Callistomys pictus (Pictet) (Rodentia, Echimyidae). A study on the geographical distribution of Callistomys pictus (Pictet, 1843) based on specimens conserved in museums, bibliography informations, and field observations is presented. The species is endemic to the Atlantic forest of the State of Bahia, Brazil. The destruction of the forests for pasture represents a serious threat to the survival of $C$. pictus and other species in southern Bahia.

KEY WORDS. Rodentia, Echimyidae, Callistomys pictus, Bahia, Brazil
\end{abstract}

O saruê-bejú, Callistomys pictus (Pictet, 1843), é um equimídeo arborícola, endêmico do Brasil, cuja distribuição geográfica se restringe à Mata Atlântica do Estado da Bahia. Trata-se de uma forma cuja pelagem exibe contrastante padrão de coloração branca e preta (Fig. 1). O comprimento da cabeça e corpo varia de 250 a $295 \mathrm{~mm}$ e a cauda de 273 a $325 \mathrm{~mm}$ (EMMONS \& VUCETICH 1998). O peso varia de 267,6 a 480 gramas $(n=3)$. As únicas informações sobre a espécie na natureza se limitam às observações feitas pelo naturalista João Moojen (MOOJEN 1952) que, para a região de Ilhéus, afirmou ser o animal "encontrado nas matas e nos cacauais".

Este trabalho aborda a distribuição geográfica de $C$. pictus no Estado da Bahia.

\section{MATERIAL E MÉTODOS}

O presente estudo é baseado em bibliografia, espécimes conservados em museus e em observações de campo realizadas pelo autor.

Com relação aos exemplares depositados em museus, estes foram objeto de inventário que envolveu 11 instituições: Centro de Pesquisas do Cacau - Comissão Executiva do Plano da Lavoura Cacaueira (CEPEC/CEPLAC), Ilhéus; Museu Nacional, Rio de Janeiro (MNRJ); Museu de Zoologia, Universidade de São Paulo (MZUSP); Muséum d'Histoire Naturelle de Genève (MHNG); Museum National d'Histoire Naturelle (MNHN), Paris; Museum Naturalis (RMNH), Leiden; Museum für Naturkunde der Humboldt, Universität zu Berlin (ZMB); Naturhistorisches Museum Wien (NMW); The Natural History Museum (BMNH), London; Universidade Estadual de Santa Cruz (ZUESC), Ilhéus e Zoologische Staatssammlung München (ZSM).

As pesquisas de campo, envolvendo observações diretas e depoimentos de moradores locais, foram desenvolvidas entre janeiro e fevereiro de 2001 nos distritos de Rio do Braço (arredores da sede distrital, fazenda Almada, Lavapés) e Aritaguá (Urucutuca), ambos pertencentes ao município de Ilhéus, Bahia.

1) Departamento de Vertebrados, Seção de Mamíferos, Museu Nacional. Quinta da Boa Vista, 20940-040 Rio de Janeiro, Rio de Janeiro, Brasil.

Revta bras. Zool. 19 (3): 631 - 635, 2002 


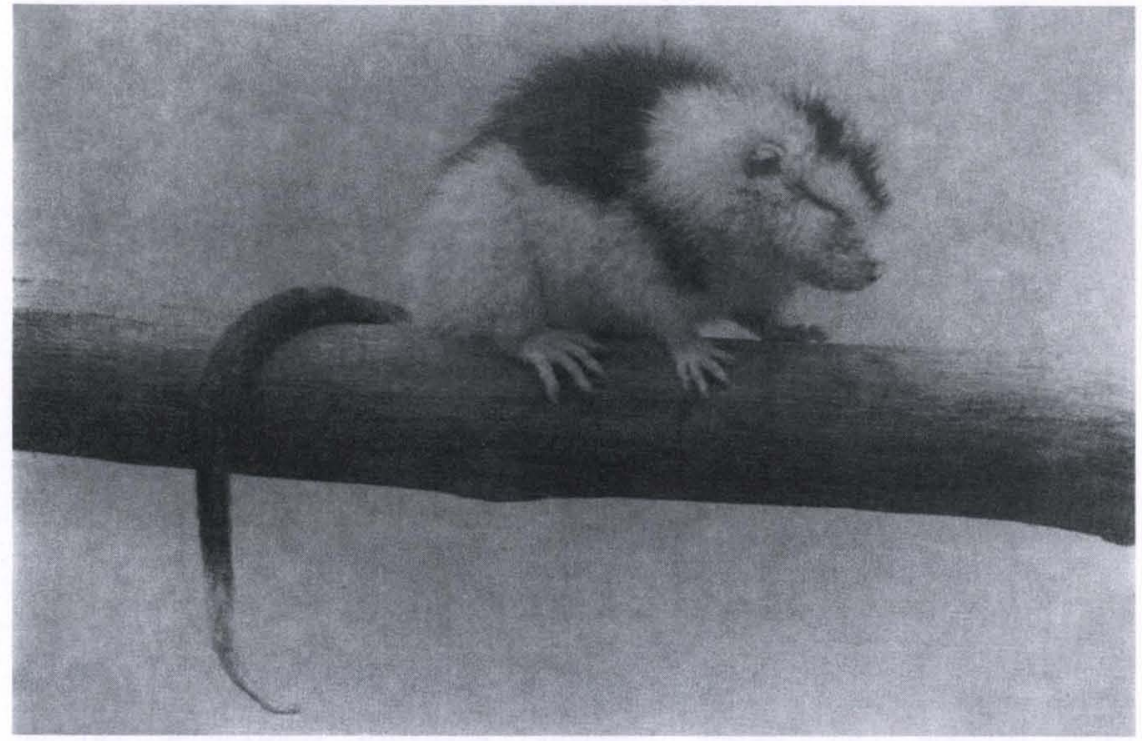

Fig. 1 Callistomys pictus, espécime fotografado pelo Dr. João Moojen, em 1944, no município de llhéus.

\section{RESULTADOS}

A espécie descrita por François Jules Pictet, a partir de um exemplar (MHNG 299.53) enviado ao Muséum d'Histoire Naturelle de Genève, por M. Blanchet, é bastante rara em coleções científicas.

O inventário envolvendo espécimes conservados detectou apenas a presença de 13 indivíduos mantidos em seis instituições (Tab. I). Ao contrário do que se imaginava, não foi possível localizar nos museus europeus indivíduos de $C$. pictus coletados pelos eminentes naturalistas e colecionadores que viveram ou visitaram a Bahia, no século XIX. É o caso de Francisco Agostinho Gomes, G.W. Freyreiss, Friedrich Sellow, Johann B. von Spix, Maximilian zu Wied Neuwied, entre outros. Os dados sobre procedência contidos nas etiquetas dos espécimes conservados no exterior são imprecisos e se limitam a "Bahia" e "South America". O exame do material conservado no Museu Nacional (MNRJ) e no Centro de Pesquisas do Cacau-Comissão Executiva do Plano da Lavoura Cacaueira (CEPEC/CEPLAC) possibilitou a tomada de medidas cranianas de alguns indivíduos que, devido a carência de informações sobre a espécie, são apresentadas na tabela II.

Entre dezembro de 1943 e abril de 1945, o então Serviço de Estudos e Pesquisas Sobre a Febre Amarela (SEPSFA) realizou investigações científicas envolvendo a captura de mamíferos silvestres (5.322 exemplares), na área dos municípios de Ilhéus e Buerarema. Na lista elaborada por LAEMMERT et al. (1946) há registro de três exemplares de "Isothrix Picta [sic] (Pictet)", os quais estão depositados no Museu Nacional (MNRJ 11207, 31545 e 31546). 
Tabela I. Relação de espécimes de Callistomys pictus conservados em coleções científicas.

\begin{tabular}{|c|c|c|c|c|}
\hline Número & Localidade & Data & Sexo & Material \\
\hline \multicolumn{5}{|l|}{ MNRJ } \\
\hline 11207 & Lavapés de Dentro, Ilhéus & 27.X.1944 & Macho & Pele e crânio \\
\hline 15453 & Ilhéus & - & Fêmea & Pele e crânio \\
\hline 31546 & llhéus & - & - & Pele \\
\hline \multicolumn{5}{|c|}{ CEPEC/CEPLAC } \\
\hline 18 & BR 415, Km22, Ilhéus & - & - & Crânio \\
\hline 24 & Fazenda Mirabela (?) Mutuns, Itabuna & 04.XII.1993 & Macho & Pele e crânio \\
\hline 28 & CEPLAC, Setor deTreinamento, Ithéus & 10.XII.1993 & Macho & Pele e crânio \\
\hline Sem número & CEPEC/CEPLAC & 1980 & - & Em álcool \\
\hline Sem número & Lomanto Júnior & 1999 & Fêmea & Em álcool \\
\hline \multicolumn{5}{|l|}{ MHNG } \\
\hline 299.53 & Bahia & - & - & Pele e crânio (holótipo) \\
\hline \multicolumn{5}{|l|}{ BMNH } \\
\hline 52.1 .5 .22 & South America & 1852 & - & Pele \\
\hline 52.6 .5 .23 & - & - & - & Crânio* \\
\hline 54.6 .5 .23 & - & - & - & Esqueleto* \\
\hline 80.9 .15 .1 & Bahia & - & - & Pele e crânio \\
\hline \multicolumn{5}{|l|}{ ZMB } \\
\hline 4809 & Bahia & - & - & Crânio \\
\hline \multicolumn{5}{|l|}{ ZUESC } \\
\hline Sem número & Fazenda Santa Maria, Ilhéus & 1986 & - & Pele \\
\hline
\end{tabular}

(*) Crânio e esqueleto pertencem ao exemplar 52.1.5.22.

Tabela II. Medidas cranianas $(\mathrm{mm}$ ) de espécimes de Callistomys pictus, de acordo com PATTON \& ROGERS (1983).

\begin{tabular}{|c|c|c|c|c|c|}
\hline \multirow{2}{*}{ Medidas } & \multicolumn{2}{|c|}{ MNRJ } & \multicolumn{3}{|c|}{ CEPEC/CEPLAC } \\
\hline & 31545 (femea) & 11207 (macho) & $18(?)$ & 24 (macho) & 28 (macho) \\
\hline Ccomprimento total & - & 61,85 & 57,34 & 55,40 & 59,65 \\
\hline Comprimento base & 47,27 & 48,20 & 46,42 & 41,72 & 47,41 \\
\hline Constrição interorbital & 14,72 & 16,65 & 12,32 & 12,60 & 13,76 \\
\hline Largura zigomática & 30,62 & 30,65 & 29,69 & 27,31 & 29,20 \\
\hline Altura crânio & 18,38 & 18,21 & 15,72 & 14,82 & 14,90 \\
\hline Comprimento bula & 12,16 & 12,57 & 12,52 & 11,68 & 13,60 \\
\hline Série molar & 11,98 & 13,30 & 12,52 & 11,91 & 13,19 \\
\hline Palatal A & 25,82 & 25,95 & 21,39 & 22,91 & 22,39 \\
\hline Palatal B & 11,46 & 13,10 & 12,01 & 12,14 & 11,87 \\
\hline Comprimento forame incisivo & 5,49 & 4,80 & 5,67 & 5,65 & 5,55 \\
\hline Comprimento nasal & - & 20,73 & 17,36 & 16,24 & 19,42 \\
\hline Comprimento rostral & - & 24,80 & 20,85 & 20,25 & 21,46 \\
\hline Altura rostro & 13,07 & 14,00 & 12,06 & 11,90 & 12,96 \\
\hline Diástema & 13,81 & 13,25 & 12,72 & 12,08 & 12,75 \\
\hline Comprimento mandibula & 35,06 & 36,45 & 33,65 & 31,10 & 33,74 \\
\hline Largura rostro & 10,78 & 11,50 & 9,82 & 9,82 & 9,81 \\
\hline
\end{tabular}

O espécime MNRJ 11207 é o único que dispõe de informação precisa sobre sua procedência. O animal foi coletado em 27 de outubro de 1944, em plantação de cacau, no lugar chamado "Lavapés de Dentro", distrito de Rio do Braço. Nas etiquetas dos outros exemplares consta apenas a referência "Ilhéus".

De outubro de 1944 até 1980, o "status" dessa espécie tornou-se uma incógnita, tendo em vista que nenhum novo exemplar foi objeto de citação na literatura ou incorporado ao acervo de algum museu. 
Foi somente a partir da preocupação de pesquisadores do Centro de Pesquisas do Cacau/CEPLAC com o impacto que representava o uso indiscriminado de iscas envenenadas para combater roedores nos cacauais que $C$. pictus voltou a ser objeto de referência (ENCARNAÇÃO et al. 1997).

As investigações envolvendo o animal permitiram levantar que ele não está restrito somente ao município de Ilhéus, pois espécimes foram encontrados em Itabuna (Mutuns - $14^{\circ} 44^{\prime} \mathrm{S}, 39^{\circ} 18^{\prime} \mathrm{W}$ ), Lomanto Junior e Elísio Medrado (Reserva Particular do Patrimônio Natural Jequitibá, Serra da Jibóia - $12^{\circ} 51$ 'S, $38^{\circ} 28^{\prime}$ 'W) (Antonia Marli V. da Encarnação, inf. pessoal). Em Ilhéus, o saruê-bejú já foi

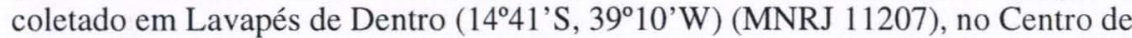
Pesquisas do Cacau/CEPLAC ( $14^{\circ} 46^{\prime}$ 'S, $\left.39^{\circ} 13^{\prime} \mathrm{W}\right)$ e na fazenda Santa Maria (14 $42^{\prime}$ S, 39 $9^{\circ} 10^{\prime} \mathrm{W}$ ) (Antonio Jorge S. Argolo, comunicação pessoal).

As pesquisas de campo permitiram verificar que o animal ocorre nos arre-

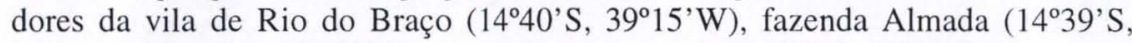
$39^{\circ} 11^{\prime} \mathrm{W}$ ) (Rui Detrei Ribeiro, com. pes.) e Lavapés (14⒊ $39^{\prime} \mathrm{S}, 39^{\circ} 11^{\prime} \mathrm{W}$ ).

Durante os estudos no distrito de Rio do Braço foi possível observar que moradores locais sabem distinguir perfeitamente o saruê-bejú de outras espécies silvestres que ocorrem na região. Isso se deve, principalmente, à coloração vistosa da pelagem do animal. Algumas pessoas admitem que eventualmente o caçam e o utilizam como alimento.

\section{DISCUSSÃo}

Os conhecimentos atuais indicam que o saruê-beju tem sua ocorrência confirmada em quatro municípios baianos (Ilhéus, Itabuna, Lomanto Junior e Elísio Medrado), mas não é improvável que espécimes venham a ser encontrados em outros locais na faixa de Mata Atlântica do Estado da Bahia.

Callistomys pictus é espécie pouco conhecida que habita uma região que vem sendo rapidamente modificada pela ação humana.

“(...) No final da década de 1940-1950, restavam da floresta original do sudeste baiano remanescentes poucos significativos, a maioria já muito alterados sob o ponto de vista ecológico, devido principalmente à ininterrupta extração seletiva secular das melhores madeiras. A maioria das matas do sudeste da Bahia é atualmente constituída de capoeiras em diferentes estágios sucessórios com apenas um ou outro trato mais idoso, parecendo aos inexperientes matas primárias, mas que na realidade não passam de autênticos capoeirões" (COIMBRA-FILHO \& CÂMARA 1996).

"A cultura do cacau tem sido, durante décadas, um dos mais importantes fatores do desenvolvimento econômico do Estado da Bahia e produto de relevo na produção agrícola da região Amazônica. No entanto, nos últimos anos, os baixos preços do produto no mercado internacional, os fatores climáticos adversos e principalmente o alastramento da doença denominada "vassoura-de-bruxa" causada pelo fungo Crinipellis perniciosa na região cacaueira baiana, levaram ao arrefecimento da cultura. Como consequência, reduziram-se em milhares de unidades os postos de trabalho e iniciou-se um intenso processo de substituição de áreas da lavoura por pastagens, principalmente, destruindo os remanescentes da mata atlântica, que eram preservados com o objetivo de proteger o cacau da exposição direta ao sol" (PINTO \& PIRES 1998).

Revta bras. Zool. 19 (3): $631-635,2002$ 
Não há, no momento, elementos suficientes que indiquem que o saruê-beju esteja em fase de extinção, entretanto, é uma espécie vulnerável que carece de proteção, já que não existe confirmação de sua ocorrência em parques nacionais ou reservas biológicas. A área do Centro de Pesquisas do Cacau/CEPLAC, com 764,68 ha, é o único local pertencente ao Poder Público, onde se tem registro confirmado da presença da espécie.

A derrubada da vegetação arbórea para a implantação ou ampliação de áreas de pastagens constitui uma séria ameaça à vida selvagem do sudeste baiano, especialmente para aquelas espécies que dependem exclusivamente da floresta para sobreviver. Neste particular inclui-se Callistomys pictus e vários outros mamíferos, inclusive alguns relacionados como em vias de extinção (BRASIL 1989), como é o caso da preguiça-de-coleira Bradypus torquatus Illiger, 1811, do mico-leão Leontopithecus chrysomelas (Kuhl, 1820) e do macaco-do-peito-amarelo Cebus xanthosternos (Wied, 1820).

AGRADECIMENTOS. Aos Doutores François J. Baud (MHNG), Jacques Cuisin (MNHN), Chris Smeenk (RMNH), Manfred Ade (ZMB), Barbara Herzig (NMW), Michael Hiermeier e Richard Kraft (ZSM) e Antônio Jorge S. Argolo (UESC) pelas informações prestadas. Ao Dr. Christopher James Tribe pelo auxílio no levantamento dos dados contidos nas etiquetas do material conservado no The Natural History Museum. À Bióloga Antonia Marli V. da Encarnação pelas facilidades para examinar os espécimes conservados no Centro de Pesquisas do Cacau/CEPLAC. Ao Sr. Washington Ferreira dos Santos pelo auxílio nos trabalhos de campo. À Dra. Leila M. Pessoa e ao Biólogo Gilson E. Iack Ximenes pela ajuda na medição dos crânios À Bibliotecária Solange P. Lyrio Gomes pelo auxílio na preparação das tabelas. Ao Dr. Ulisses Caramaschi pela leitura do texto e sugestões.

\section{REFERÊNCIAS BIBLIOGRÁFICAS}

Brasil. 1989. Lista Oficial das Espécies da Fauna Brasileira Ameaçada de Extinção. Brasília, IBAMA, Portaria no. 1522, de 19 de dezembro de 1989, Diário Oficial da União, 22 de dezembro de 1989.

Colmbra-Filho, A.F. \& I.G. CÂmara. 1996. Os Limites Originais do Bioma Mata Atlântica na Região Nordeste. Rio de Janeiro, Fundação Brasileira para a Conservação da Natureza, 86p.

Emmons, L.H \& M.G. VucETich. 1998. The identity of Winge's Lasiuromys villosus and the description of a new genus of echimyid rodent (Rodentia: Echimyidae). Amer. Mus. Nov. 3223: 1-11.

Encarnação, A.M.V.; A.J.S. Argolo \& B.S. Santos. 1997. Em risco de extinção: Emprego de iscas raticidas nos cacauais ameaça roedor raro no sul da Bahia. Ci. Hoje 22 (130): 54.

LAEMMERT JR., H.W.; L.C. FERREIRA \& R.M. TAYLOR. 1946. An epidemiological study of jungle yellow fever in an endemic area in Brazil. Part II. Investigation of vertebrate hosts and arthropod vectors. Amer. Jour. Trop. Med. Suppl. 26 (6): 1-69.

Moojen, J. 1952. Os Roedores do Brasil. Rio de Janeiro, Ministério da Educação e Saúde, Instituto Nacional do Livro, 214p.

PATTON, J.L. \& M.A. Rogers. 1983. Systematic implications of non geographic variation in the spiny rat genus Proechimys (Echimyidae). Z. Saugetierkunde 48: 363-370.

PICTET, F.J. 1843. Second notice sur les animaux nouveaux on peu connus du Musée de Genève. Mem. Soc. Phys. Hist. Nat. Genève 10 (1): 201-213.

PINTO, L.R.M. \& J.L. PIRES. 1998. Seleção de plantas de cacau resistentes à vassoura de bruxa. Bol. Téc. CEPLAC/CEPEC, Ilhéus, 181: 1-35

Recebido em 10.VIII.2001; aceito em 11.VII.2002. 